\title{
Solution of Temperature Distribution in a Radiating Fin Using Homotopy Perturbation Method
}

\author{
M. J. Hosseini, M. Gorji, and M. Ghanbarpour \\ Department of Mechanical Engineering, Noshirvani University of Technology, P.O. Box 484, Babol, Iran \\ Correspondence should be addressed to M. Gorji, gorji@nit.ac.ir
}

Received 27 November 2008; Accepted 22 January 2009

Recommended by Saad A. Ragab

Radiating extended surfaces are widely used to enhance heat transfer between primary surface and the environment. The present paper applies the homotopy perturbation to obtain analytic approximation of distribution of temperature in heat fin radiating, which is compared with the results obtained by Adomian decomposition method (ADM). Comparison of the results obtained by the method reveals that homotopy perturbation method (HPM) is more effective and easy to use.

Copyright (C) 2009 M. J. Hosseini et al. This is an open access article distributed under the Creative Commons Attribution License, which permits unrestricted use, distribution, and reproduction in any medium, provided the original work is properly cited.

\section{Introduction}

Most scientific problems and phenomena such as heat transfer occur nonlinearly. Except a limited number of these problems, it is difficult to find the exact analytical solutions for them. Therefore, approximate analytical solutions are searched and were introduced [1-5], among which homotopy perturbation method (HPM) [6-12] and Adomain decomposition method $(\mathrm{ADM})[13,14]$ are the most effective and convenient ones for both weakly and strongly nonlinear equations.

The analysis of space radiators, frequently provided in published literature, for example, [15-22], is based upon the assumption that the thermal conductivity of the fin material is constant. However, since the temperature difference of the fin base and its tip is high in the actual situation, the variation of the conductivity of the fin material should be taken into consideration and includes the effects of the variation of the thermal conductivity of the fin material. The present analysis considers the radiator configuration shown in Figure 1. In the design, parallel pipes are joined by webs, which act as radiator fins. Heat flows by conduction from the pipes down the fin and radiates from both surfaces. 


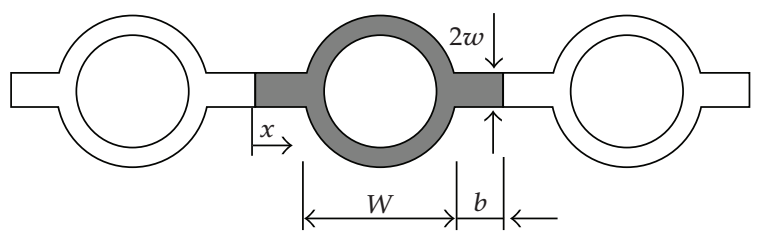

Figure 1: Schematic of a heat fin radiating element.

Here, the fin problem is solved to obtain the distribution of temperature of the fin by homotopy perturbation method and compared with the result obtained by the Adomian decomposition method, which is used for solving various nonlinear fin problems [23-25].

\section{The Fin Problem}

A typical heat pipe space radiator is shown in Figure 1. Both surfaces of the fin are radiating to the vacuum of outer space at a very low temperature, which is assumed equal to zero absolute. The fin is diffuse-grey with emissivity $\varepsilon$, and has temperature-dependent thermal conductivity $k$, which depends on temperature linearly. The base temperature $T_{b}$ of the fin and tube surfaces temperature is constant; the radiative exchange between the fin and heat pipe is neglected. Since the fin is assumed to be thin, the temperature distribution within the fin is assumed to be one-dimensional. The energy balance equation for a differential element of the fin is given as [26]:

$$
2 w \frac{d}{d x}\left[k(T) \frac{d T}{d x}\right]-2 \varepsilon \sigma T^{4}=0,
$$

where $k(T)$ and $\sigma$ are the thermal conductivity and the Stefan-Boltzmann constant, respectively. The thermal conductivity of the fin material is assumed to be a linear function of temperature according to

$$
K(T)=K_{b}\left[1+\lambda\left(T-T_{b}\right)\right],
$$

where $k_{b}$ is the thermal conductivity at the base temperature of the fin and $\lambda$ is the slope of the thermal conductivity temperature curve.

Employing the following dimensionless parameters:

$$
\theta=\frac{T}{T_{b}}, \quad \psi=\frac{\varepsilon \sigma b^{2} T_{b}^{3}}{k w}, \quad \xi=\frac{x}{b}, \quad \beta=\lambda T_{b},
$$

the formulation of the fin problem reduces to

$$
\frac{d^{2} \theta}{d \xi^{2}}+\beta\left(\frac{d \theta}{d \xi}\right)^{2}+\beta \theta \frac{d^{2} \theta}{d \xi^{2}}-\psi \theta^{4}=0,
$$


with boundary conditions

$$
\begin{aligned}
& \frac{d \theta}{d \xi}=0 \quad \text { at } \xi=0, \\
& \theta=1 \quad \text { at } \xi=1 .
\end{aligned}
$$

\section{Basic Idea of Homotopy Perturbation Method}

In this study, we apply the homotopy perturbation method to the discussed problems. To illustrate the basic ideas of the method, we consider the following nonlinear differential equation,

$$
A(\theta)-f(r)=0
$$

where $A(\theta)$ is defined as follows:

$$
A(\theta)=L(\theta)+N(\theta)
$$

where $L$ stands for the linear and $N$ for the nonlinear part. Homotopy perturbation structure is shown as the following equation:

$$
H(\theta, P)=(1-p)\left[L(\theta)-L\left(\theta_{0}\right)\right]+p[A(\theta)-f(r)]=0
$$

Obviously, using (3.3) we have

$$
\begin{aligned}
& H(\theta, 0)=L(\theta)-L\left(\theta_{0}\right)=0, \\
& H(\theta, 1)=A(\theta)-f(r)=0,
\end{aligned}
$$

where $p \in[0,1]$ is an embedding parameter and $\theta_{0}$ is the first approximation that satisfies the boundary condition. We Consider $\theta$ and as

$$
\theta=\sum_{i=0}^{M} p^{i} \theta_{i}=\theta_{0}+p \theta_{1}+p^{2} \theta_{2}+p^{3} \theta_{3}+p^{4} \theta_{4}+\cdots
$$

\section{The Fin Temperature Distribution}

Following homotopy-perturbation method to (2.4a), (2.4b), and (2.4c), linear and non-linear parts are defined as

$$
\begin{aligned}
L(\theta) & =\frac{d^{2} \theta}{d \xi^{2}}, \\
N(\theta) & =\beta\left(\frac{d \theta}{d \xi}\right)^{2}+\beta \theta \frac{d^{2} \theta}{d \xi^{2}}-\psi \theta^{4},
\end{aligned}
$$

with the boundary condition given in (2.4b), $\theta(0)$ is any arbitrary constant, $C$. 
Then we have

$$
\frac{d \theta}{d \xi}=0 \quad \text { at } \xi=0, \quad \theta=C \quad \text { at } \xi=0
$$

Substituting (3.5) in to (4.1) and then into (3.3) and rearranging based on power of $p$-terms, we have the following

$\left(p^{0}\right)$

$$
\begin{gathered}
\frac{\partial^{2}}{\partial \xi^{2}} \theta_{0}(\xi)=0, \\
\frac{d \theta_{0}}{d \xi}=0 \quad \text { at } \xi=0, \quad \theta_{0}=C \quad \text { at } \xi=0
\end{gathered}
$$

$\left(p^{1}\right)$

$$
\begin{gathered}
\left(\frac{\partial^{2}}{\partial \xi^{2}} \theta_{1}(\xi)\right)+\beta \theta_{0}(\xi)\left(\frac{\partial^{2}}{\partial \xi^{2}} \theta_{0}(\xi)\right)+\beta\left(\frac{\partial}{\partial \xi} \theta_{0}(\xi)\right)^{2}-\psi \theta_{0}^{4}(\xi)=0, \\
\frac{d \theta_{1}}{d \xi}=0 \quad \text { at } \xi=0, \quad \theta_{1}=0 \quad \text { at } \xi=0 ;
\end{gathered}
$$

$\left(p^{2}\right)$

$$
\begin{gathered}
\left(\frac{\partial^{2}}{\partial \xi^{2}} \theta_{2}(\xi)\right)+\beta \theta_{0}(\xi)\left(\frac{\partial^{2}}{\partial \xi^{2}} \theta_{1}(\xi)\right)+\beta \theta_{1}(\xi)\left(\frac{\partial^{2}}{\partial \xi^{2}} \theta_{0}(\xi)\right) \\
+2 \beta\left(\frac{\partial}{\partial \xi} \theta_{0}(\xi)\right)\left(\frac{\partial}{\partial \xi} \theta_{1}(\xi)\right)-4 \psi \theta_{0}^{3}(\xi) \theta_{1}(\xi)=0, \\
\frac{d \theta_{2}}{d \xi}=0 \quad \text { at } \xi=0, \quad \theta_{2}=0 \quad \text { at } \xi=0 ;
\end{gathered}
$$

$\left(p^{3}\right)$

$$
\begin{gathered}
\left(\frac{\partial^{2}}{\partial \xi^{2}} \theta_{3}(\xi)\right)+\beta \theta_{2}(\xi)\left(\frac{\partial^{2}}{\partial \xi^{2}} \theta_{0}(\xi)\right)+\beta \theta_{0}(\xi)\left(\frac{\partial^{2}}{\partial \xi^{2}} \theta_{2}(\xi)\right)+\beta\left(\frac{\partial}{\partial \xi} \theta_{1}(\xi)\right)^{2} \\
+\beta \theta_{1}(\xi)\left(\frac{\partial^{2}}{\partial \xi^{2}} \theta_{1}(\xi)\right)+2 \beta\left(\frac{\partial}{\partial \xi} \theta_{0}(\xi)\right)\left(\frac{\partial}{\partial \xi} \theta_{2}(\xi)\right)-4 \psi \theta_{0}^{3}(\xi) \theta_{2}(\xi) \\
-6 \psi \theta_{0}^{2}(\xi) \theta_{1}^{2}(\xi)=0, \\
\frac{d \theta_{3}}{d \xi}=0 \quad \text { at } \xi=0, \quad \theta_{3}=0 \quad \text { at } \xi=0
\end{gathered}
$$




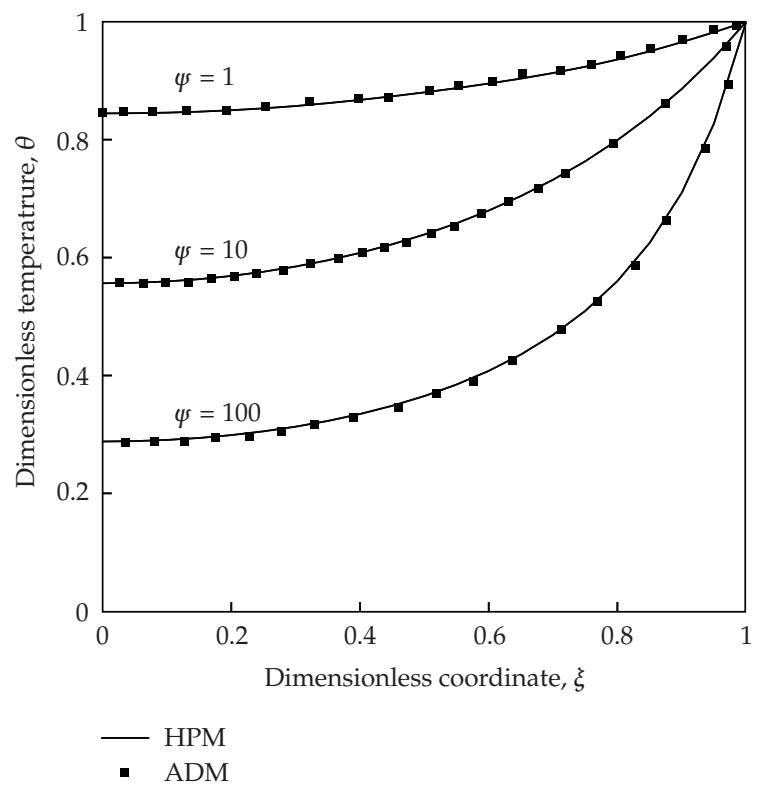

Figure 2: Comparison of the HPM and ADM for $\beta=1.0$ and $M=14$.

$\left(p^{4}\right)$

$$
\begin{gathered}
\left(\frac{\partial^{2}}{\partial \xi^{2}} \theta_{4}(\xi)\right)+\beta \theta_{3}(\xi)\left(\frac{\partial^{2}}{\partial \xi^{2}} \theta_{0}(\xi)\right)+\beta \theta_{0}(\xi)\left(\frac{\partial^{2}}{\partial \xi^{2}} \theta_{3}(\xi)\right)-4 \psi \theta_{0}^{3}(\xi) \theta_{3}(\xi) \\
+\beta \theta_{2}(\xi)\left(\frac{\partial^{2}}{\partial \xi^{2}} \theta_{1}(\xi)\right)+2 \beta\left(\frac{\partial}{\partial \xi} \theta_{0}(\xi)\right)\left(\frac{\partial}{\partial \xi} \theta_{3}(\xi)\right)-12 \psi \theta_{0}^{2}(\xi) \theta_{2}(\xi) \theta_{1}(\xi) \\
+\beta\left(\frac{\partial^{2}}{\partial \xi^{2}} \theta_{1}(\xi)\right)\left(\frac{\partial}{\partial \xi} \theta_{2}(\xi)\right)+\beta \theta_{1}(\xi)\left(\frac{\partial^{2}}{\partial \xi^{2}} \theta_{2}(\xi)\right)-4 \psi \theta_{0}(\xi) \theta_{1}^{3}(\xi)=0 \\
\frac{d \theta_{4}}{d \xi}=0 \quad \text { at } \xi=0, \quad \theta_{4}=0 \quad \text { at } \xi=0
\end{gathered}
$$

and so forth.

By increasing the number of the terms in the solution, higher accuracy will be obtained. Since the remaining terms are too long to be mentioned in here, the results are shown in tables.

Solving (4.3a), (4.4a), (4.5a), (4.6a), and (4.7a) results in $\theta(\xi)$. When $p \rightarrow 1$, we have

$$
\begin{aligned}
\theta(\xi)= & C+\frac{1}{2} \psi C^{4} \xi^{2}+\frac{1}{6} \psi^{2} C^{7} \xi^{4}-\frac{1}{2} \beta C^{5} \psi \xi^{2}+\frac{13}{180} \psi^{3} C^{10} \xi^{6} \\
& -\frac{11}{24} \psi^{2} C^{8} \beta \xi^{4}+\frac{1}{2} \beta^{2} C^{6} \psi \xi^{2}+\frac{23}{720} \psi^{4} C^{13} \xi^{8}-\frac{19}{60} \psi^{3} C^{11} \beta \xi^{6} \\
& +\frac{7}{8} \beta^{2} C^{9} \psi^{2} \xi^{4}-\frac{1}{2} \beta^{3} C^{7} \psi \xi^{2} \cdots
\end{aligned}
$$


Table 1: The dimensionless tip temperature for $\psi=1$.

\begin{tabular}{lcccccccc}
\hline $\begin{array}{l}\text { Number of the terms } \\
\text { in the solution }(M)\end{array}$ & \multicolumn{2}{c}{$M=5$} & \multicolumn{2}{c}{$M=7$} & \multicolumn{2}{c}{$M=10$} & \multicolumn{2}{c}{$M=14$} \\
\hline Method & HPM & ADM & HPM & ADM & HPM & ADM & HPM & ADM \\
\hline$\beta=0.6$ & 0.827821 & 0.819185 & 0.826552 & 0.825079 & 0.8267319 & 0.825063 & 0.82675 & 0.825052 \\
$\beta=0.4$ & 0.813389 & 0.814489 & 0.813351 & 0.813279 & 0.8133683 & 0.810866 & 0.813369 & 0.813236 \\
$\beta=0.2$ & 0.797708 & 0.80021 & 0.797712 & 0.799025 & 0.7977122 & 0.797812 & 0.797712 & 0.797809 \\
$\beta=0$ & 0.779177 & 0.777778 & 0.779147 & 0.777765 & 0.7791452 & 0.776554 & 0.779145 & 0.775333 \\
$\beta=-0.2$ & 0.757702 & 0.752987 & 0.75698 & 0.752967 & 0.7568182 & 0.754144 & 0.756802 & 0.754132 \\
$\beta=-0.4$ & 0.819743 & 0.814465 & 0.816013 & 0.813252 & 0.8132926 & 0.810831 & 0.812155 & 0.813201 \\
$\beta=-0.6$ & 0.710294 & 0.715063 & 0.703219 & 0.700812 & 0.6988481 & 0.696134 & 0.696764 & 0.694918 \\
\hline
\end{tabular}

Table 2: The dimensionless tip temperature for $\psi=1000$.

\begin{tabular}{lcccccccc}
\hline $\begin{array}{l}\text { Number of the terms } \\
\text { in the solution }(M)\end{array}$ & \multicolumn{2}{c}{$M=5$} & \multicolumn{2}{c}{$M=7$} & \multicolumn{2}{c}{$M=10$} & \multicolumn{2}{c}{$M=14$} \\
\hline Method & HPM & ADM & HPM & ADM & HPM & ADM & HPM & ADM \\
\hline$\beta=0.6$ & 0.139382 & 0.144312 & 0.135502 & 0.137711 & 0.1330265 & 0.134125 & 0.132616 & 0.132718 \\
$\beta=0.4$ & 0.138351 & 0.143412 & 0.134243 & 0.136526 & 0.1315594 & 0.132756 & 0.130091 & 0.131026 \\
$\beta=0.2$ & 0.137341 & 0.142026 & 0.133009 & 0.134937 & 0.1301168 & 0.131213 & 0.129486 & 0.129613 \\
$\beta=0$ & 0.136349 & 0.141055 & 0.131797 & 0.133785 & 0.1286995 & 0.129663 & 0.127504 & 0.127327 \\
$\beta=-0.2$ & 0.135376 & 0.140165 & 0.13061 & 0.132654 & 0.127308 & 0.128143 & 0.125348 & 0.125431 \\
$\beta=-0.4$ & 0.134422 & 0.139565 & 0.129445 & 0.131856 & 0.1259426 & 0.127342 & 0.124118 & 0.124432 \\
$\beta=-0.6$ & 0.133485 & 0.138374 & 0.128303 & 0.130525 & 0.1246034 & 0.125336 & 0.122715 & 0.122936 \\
\hline
\end{tabular}

\section{Results}

The coefficient $C$ representing the temperature at the fin tip can be evaluated from the boundary condition given in (2.4c) using the numerical method.

Tables 1 and 2 show the dimensionless tip temperature, that is, coefficient $C$, for different thermal conductivity parameters, $\beta$. The tables state that the convergence of the solution for the higher thermo-geometric fin parameter, $\psi$, is faster than the solution with lower fin parameter. It is clear from the tables that the solution is convergent. In order to investigate the accuracy of the homotopy solution, the problem is compared with decomposition solution [26], also the corresponding results are presented in Figure 2. It should be mentioned that the homotopy results in the tables are arranged for first 5, 7, 10, 14 terms of the solution $(M)$. It is seen that the results by homotopy perturbation method, and adomian decomposition method are in good agreement. The results of the comparison show that the difference is $3.1 \%$ in the case of the strongest nonlinearity, that is, $\beta=1.0$ and $\psi=100$.

\section{Conclusions}

In this work, homotopy perturbation method has been successfully applied to a typical heat pipe space radiator. The solution shows that the results of the present method are in excellent agreement with those of ADM and the obtained solutions are shown in the figure 
and tables. Some of the advantage of HPM are that reduces the volume of calculations with the fewest number of iterations, it can converge to correct results. The proposed method is very simple and straightforward. In our work, we use the Maple Package to calculate the functions obtained from the homotopy perturbation method.

\section{References}

[1] E. M. Abulwafa, M. A. Abdou, and A. A. Mahmoud, "The solution of nonlinear coagulation problem with mass loss," Chaos, Solitons E Fractals, vol. 29, no. 2, pp. 313-330, 2006.

[2] A. Aziz and T. Y. Na, Perturbation Methods in Heat Transfer, Series in Computational Methods in Mechanics and Thermal Sciences, Hemisphere, Washington, DC, USA, 1984.

[3] J.-H. He, "Some asymptotic methods for strongly nonlinear equations," International Journal of Modern Physics B, vol. 20, no. 10, pp. 1141-1199, 2006.

[4] M. M. Rahman and T. Siikonen, "An improved simple method on a collocated grid," Numerical Heat Transfer Part B, vol. 38, no. 2, pp. 177-201, 2000.

[5] A. V. Kuznetsov and K. Vafai, "Comparison between the two- and three-phase models for analysis of porosity formation in aluminum-rich castings," Numerical Heat Transfer Part A, vol. 29, no. 8, pp. 859-867, 1996.

[6] J.-H. He, "Application of homotopy perturbation method to nonlinear wave equations," Chaos, Solitons \& Fractals, vol. 26, no. 3, pp. 695-700, 2005.

[7] J.-H. He, "Homotopy perturbation method for bifurcation of nonlinear problems," International Journal of Nonlinear Sciences and Numerical Simulation, vol. 6, no. 2, pp. 207-208, 2005.

[8] J.-H. He, "Homotopy perturbation technique," Computer Methods in Applied Mechanics and Engineering, vol. 178, no. 3-4, pp. 257-262, 1999.

[9] J.-H. He, "Variational iteration method for autonomous ordinary differential systems," Applied Mathematics and Computation, vol. 114, no. 2-3, pp. 115-123, 2000.

[10] J.-H. He, "A coupling method of a homotopy technique and a perturbation technique for non-linear problems," International Journal of Non-Linear Mechanics, vol. 35, no. 1, pp. 37-43, 2000.

[11] J.-H. He, "Homotopy perturbation method: a new nonlinear analytical technique," Applied Mathematics and Computation, vol. 135, no. 1, pp. 73-79, 2003.

[12] J.-H. He, "The homotopy perturbation method for nonlinear oscillators with discontinuities," Applied Mathematics and Computation, vol. 151, no. 1, pp. 287-292, 2004.

[13] G. Adomian, "A review of the decomposition method in applied mathematics," Journal of Mathematical Analysis and Applications, vol. 135, no. 2, pp. 501-544, 1988.

[14] G. Adomian, Solving Frontier Problems of Physics: The Decomposition Method, vol. 60 of Fundamental Theories of Physics, Kluwer Academic Publishers, Dordrecht, The Netherlands, 1994.

[15] J. G. Bartas and W. H. Sellers, "Radiation fin effectiveness," Journal of Heat Transfer, vol. 82C, pp. 73-75, 1960.

[16] J. E. Wilkins Jr., "Minimizing the mass of thin radiating fins," Journal of Aerospace Science, vol. 27, pp. 145-146, 1960.

[17] B. V. Karlekar and B. T. Chao, "Mass minimization of radiating trapezoidal fins with negligible base cylinder interaction," International Journal of Heat and Mass Transfer, vol. 6, no. 1, pp. 33-48, 1963.

[18] R. D. Cockfield, "Structural optimization of a space radiator," Journal of Spacecraft Rockets, vol. 5, no. 10, pp. 1240-1241, 1968.

[19] H. Keil, "Optimization of a central-fin space radiator," Journal of Spacecraft Rockets, vol. 5, no. 4, pp. 463-465, 1968.

[20] B. T. F. Chung and B. X. Zhang, "Optimization of radiating fin array including mutual irradiations between radiator elements," Journal of Heat Transfer, vol. 113, no. 4, pp. 814-822, 1991.

[21] C. K. Krishnaprakas and K. B. Narayana, "Heat transfer analysis of mutually irradiating fins," International Journal of Heat and Mass Transfer, vol. 46, no. 5, pp. 761-769, 2003.

[22] R. J. Naumann, "Optimizing the design of space radiators," International Journal of Thermophysics, vol. 25, no. 6, pp. 1929-1941, 2004.

[23] C. H. Chiu and C. K. Chen, "A decomposition method for solving the convective longitudinal fins with variable thermal conductivity," International Journal of Heat and Mass Transfer, vol. 45, no. 10, pp. 2067-2075, 2002.

[24] C. H. Chiu and C. K. Chen, "Application of Adomian's decomposition procedure to the analysis of convective-radiative fins," Journal of Heat Transfer, vol. 125, no. 2, pp. 312-316, 2003. 
[25] D. Lesnic and P. J. Heggs, "A decomposition method for power-law fin-type problems," International Communications in Heat and Mass Transfer, vol. 31, no. 5, pp. 673-682, 2004.

[26] C. Arslanturk, "Optimum design of space radiators with temperature-dependent thermal conductivity," Applied Thermal Engineering, vol. 26, no. 11-12, pp. 1149-1157, 2006. 


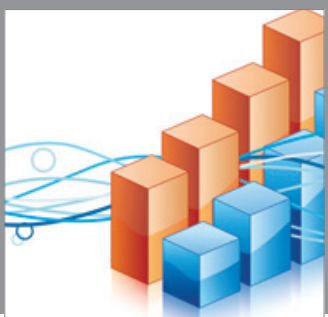

Advances in

Operations Research

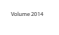

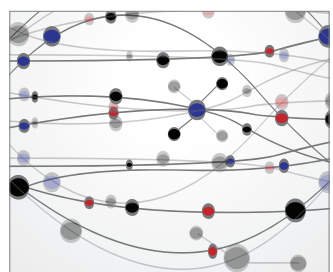

\section{The Scientific} World Journal
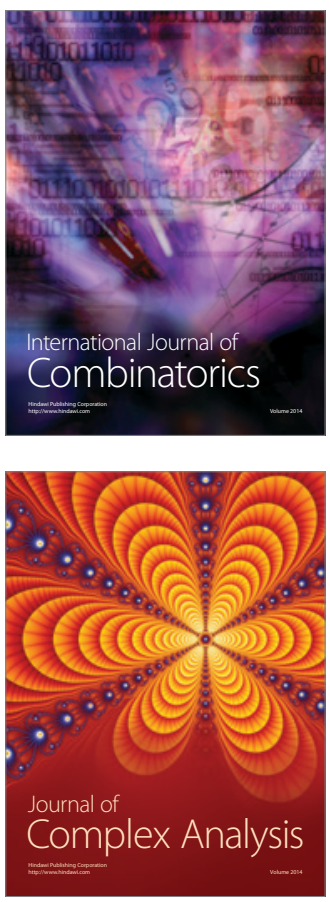

International Journal of

Mathematics and

Mathematical

Sciences
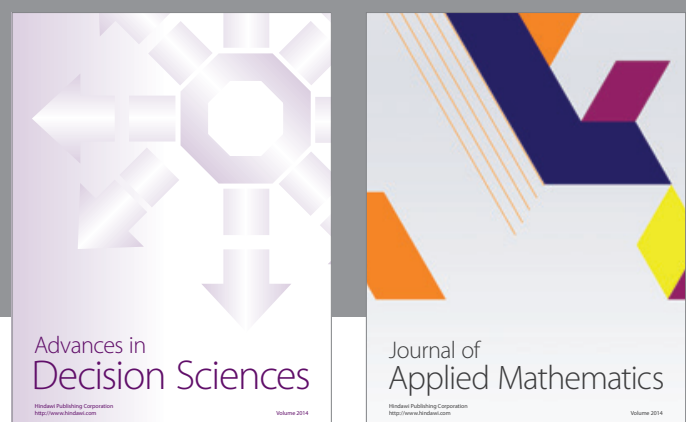

Journal of

Applied Mathematics
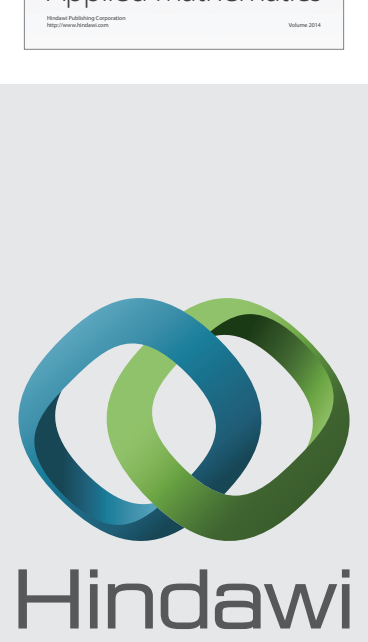

Submit your manuscripts at http://www.hindawi.com
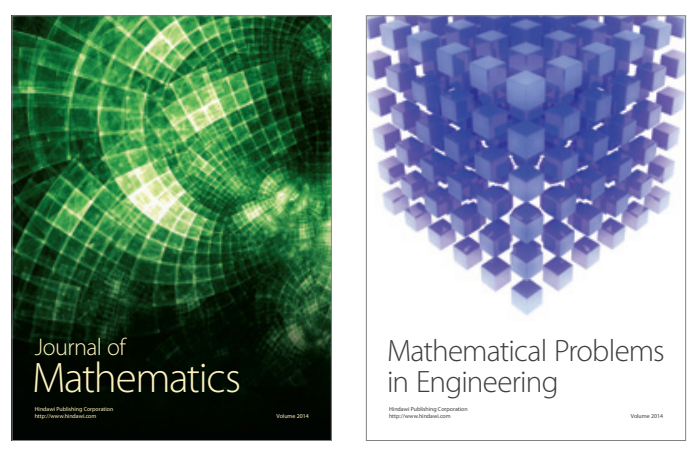

Mathematical Problems in Engineering
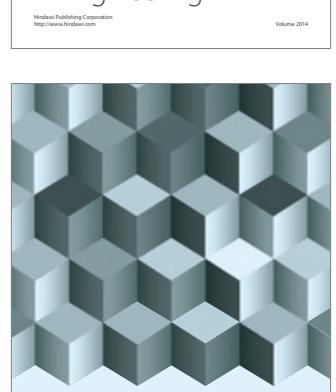

Journal of

Function Spaces
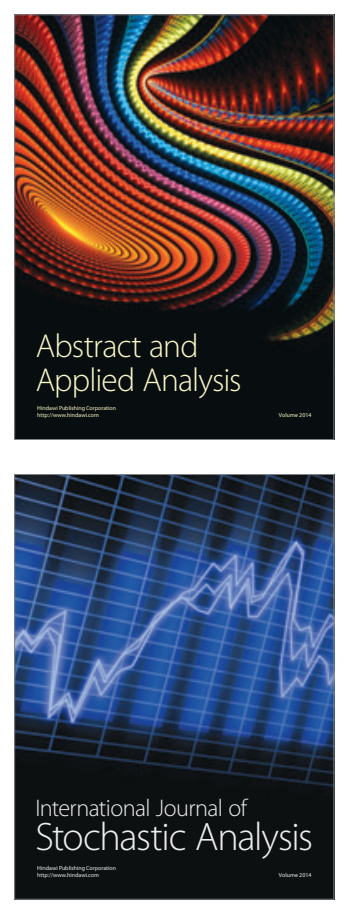

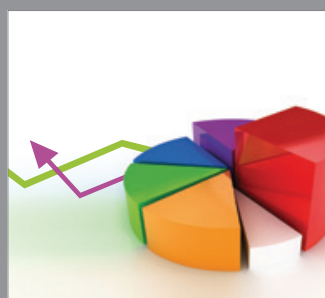

ournal of

Probability and Statistics

Promensencen
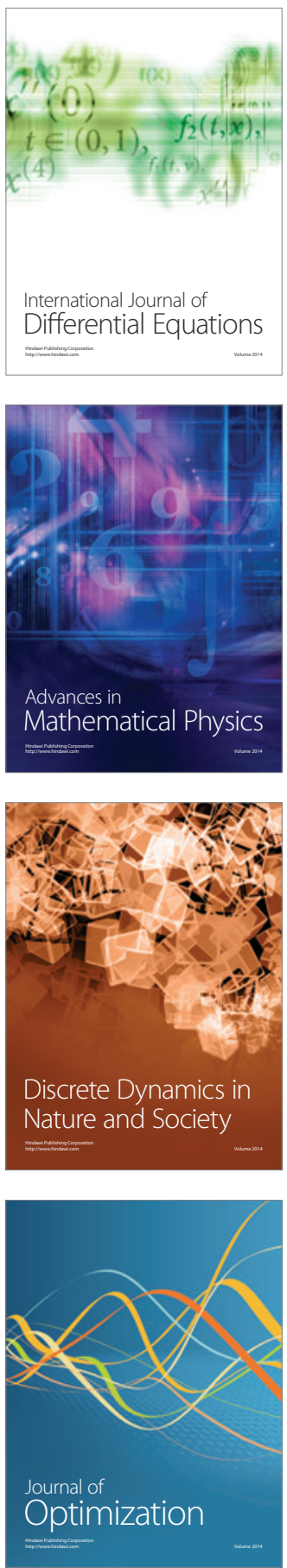